\title{
Research and Design No Light Spot in Front Located Hysteroscopy
}

\author{
Kun Dai ${ }^{1, a}$, Bo Xiang ${ }^{2}$ and Zhaomin $\mathrm{Jia}^{2}$ \\ ${ }^{1}$ Electrical Department, Tangshan Polytechnic College , Tangshan, 063020, china \\ ${ }^{2}$ Intelligence and Information school of Tangshan University, Tangshan 063020, Heibei, P.R.China
}

\begin{abstract}
This paper first introduces the recent development of the popular hysteroscopy, the international minimally invasive diagnosis instrument, and then analyzes the lighting and video of hysteroscopy. According to high energy consumption of common hysteroscopy, front located hysteroscopy system is put forward. The no light spot principles, the lens structure, the manufacture process of lens prototype, the trial manufacturing process of power box are all discussed in this paper. It is the first time that prototype has been design and tested in domestic. In the normal conditions, the energy consumption decreased by $298 / 300$. And the volume, the weight, and the price of the designed front located hysteroscopy are greatly reduced. Moreover, tests show that it can apply to special conditions such as the disaster relief field and field operation.
\end{abstract}

\section{Introduction}

Hysteroscopy is the advanced minimally invasive medical instrument [1], used for examination and treatment of gynecology uterine cavity. In October, 2010, the 64th session of China international medical equipment exposition, numerous hysteroscopy produced by Chinese and foreign manufacturers are exhibited. In spite of the different in manufacturer and brands, the working principle and composition are the same. The working principle and composition of hysteroscopy are shown in figure 1, the front of the lens body is inserted to the uterine and enlarge the to-be-observed parts. Because of its intuitive and preciseness, it becomes the first choice in examining the gynecological hemorrhagic diseases and intrauterine lesions. It is a kind of fiber illuminant endoscope, mainly including chamber lens, energy system, illuminant systems and imaging system.

A $300 \mathrm{~W}$ xenon lamp is used as the power source. Light is transmitted by optical fiber and the lighting is realized by the light of the conduction body. Image can be gained through imaging system by light transmitting to cavity via video conversion on the monitor. Energy systems provide energy for the light source system and the imaging system. The combination device has a characteristic of high power consumption, big volume, high price and rigid using environment. Researches show: light shall finally focused on local lighting of the irradiated content, light loss in optical cable and optical transmission conduction; the long transmission distance by light transmission to cavity lens imaging also brings certain loss. Therefore, the front located hysteroscopy is first developed and tested, and the effect is satisfactory.

\footnotetext{
a Corresponding author: daikun943@126.com
} 


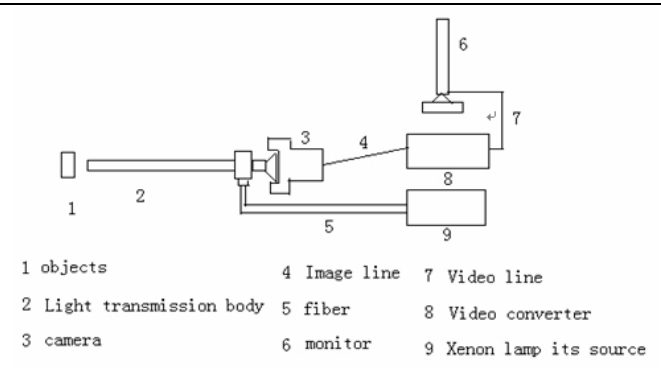

Figure 1. Hysteroscopy principle diagram

\section{Structure of the no light spot front located hysteroscopy system}

A. The Principle Of No Light Spot Front Located Hysteroscopy System

The principle of No Light Spot front located hysteroscopy system is shown in figure 2.

B. By installing the camera and lighting together, directly lighting and videoing on the subject of local, decoding through the link of guide bar, connection box at the tail and the 4 core connecting plug, linking to the power box with the wire, realizing the power source lighting and video signal transmission, video signals are transmitted by the power box through video line and being displayed as the image on the monitor. The tail connecting box is functioned as a handle to change the angle and distance between the camera and the subject to be lighted in order to get the best perturbation state. Under appropriate conditions, the connecting box can be used to fix or support something.

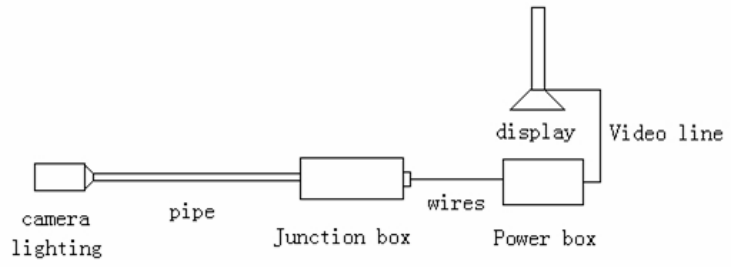

Figure 2. New hysteroscopy system

C. The Constitute of the Front Located Hysteroscopy Lens

The constitute of the front located hysteroscopy lens is shown in figure 3.On the left, it is the face Schematic. And on the right of Figure 3, it is the side schematic diagram. The light source systems adopt the six HLEDs to realize the lighting [2] [3] [4]. The total power of luminous is less than $0.5 \mathrm{~W}$. The cavity lens adopt a $\varphi 2 \mathrm{~mm}$ high-definition camera and a $\varphi 3.5 \mathrm{~mm}$ decoding video circuit. A $0.2 \mathrm{~mm}$ crystal abrasive disc is used to protect the video circuitry. All of the devices are installed in a space with $\varphi 5 \mathrm{~mm}$ and $15 \mathrm{~mm}$-long, The silver line which can be resistant to high temperature is used to draw the control video out with 2 piece of wires. one is for videoing, and the other one is for lighting.

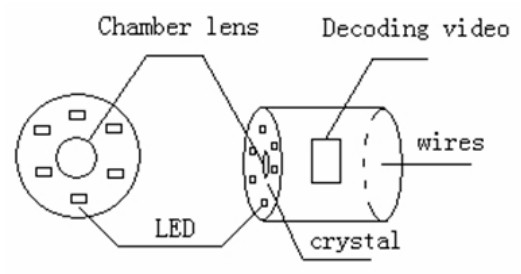

Figure 3. Front hysteroscopy lens 
D. The Constitute of no light spot the Front Located Hysteroscopy Lens

The constitute of the no light spot front located hysteroscopy lens is shown in figure 4.

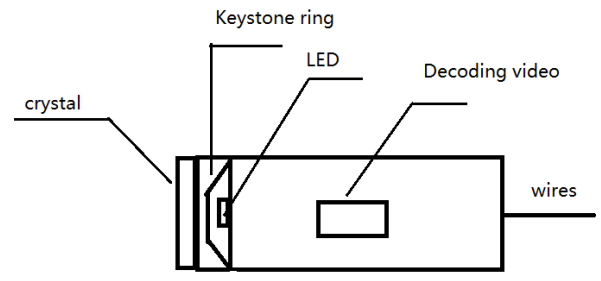

Figure 4. No light spot the Front Located Hysteroscopy Lens

After the test, Keystone ring Angle is 5 degrees.

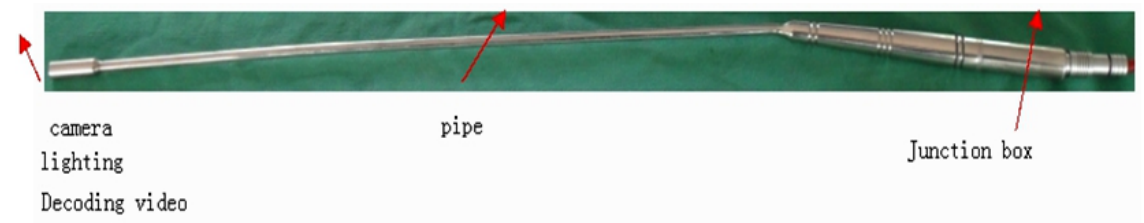

Figure 5. Camera shell

\section{No light spot front hysteroscopy system trial-production process}

\section{A. Lens Prototype Trial Producing Process}

1. Test the parameter of the voltage current of LED [2],[4], and measure the working properties, determine the control parameters, control scheme and connection schemes based on the special requirements of surgical instruments.

2. parameters, control scheme and connection schemes based on the special requirements of surgical instruments. Determine cameras and decoding working circuit voltage range, also combined with surgical instruments special requirements to determine control parameters, control scheme.

3. Manufacture the camera shell in machining method, welding method as shown in Figure 5

4. Weld clear cameras and decoding circuit with the power line, video line. Weld six miniature HLEDs and draw the lighting line, the length of the wire should be exceeded the connection plug tail $10 \mathrm{~mm}$.

5. Make the camera and LED lead on the left and thread through the steel body on the right. The wire should be through the right and until to the tail part.

6. Detect the working situation of the cameras and the LED. If it is normal, go to the next step.

7. Fix the cameras, the LEDs and the decoder circuit.

8. Test circuit.

9. Fix crystal chips.

10. Install and fix the connecting plugs

$B$. Trial Production of Power Box

The constitute of the power box as shown in Figure6.

1. Design and install the camera circuit and the LED control circuit using the multi-function PCB with 30mm-wide and 50mm-long [5] [6]. By measuring parameters through the instrument, their parameters should be adjusted to correspondent with the need of lens prototype. 
2. Punch two holes at the front and back of the power box, respectively, and install the power switch, the plug implement, the LED which can display the electric power, the video interface, and the charging interface.

3. Connect the LED with the circuit which can display the low voltage.

4. Fix the 12V high-power Li-ion batteries and multifunctional PCB.

5. Connect the plug sensor and the multi-function circuit. Connect the circuit between the battery and the low voltage circuit. Connect the circuit between the video joint and the multifunction PCB. Connect the circuit between the charging interface and the battery. Connecting the circuit between the battery and multi-functional PCB.

6. Coverer and charge.

\section{Realization of no light spot front located hysteroscopy camera and its effect}

Disinfect the lens prototype and attachment with formalin, connect lens prototype and power box with wire, and connect power box and monitor with video cable. Adapt the monitor with $12 \mathrm{v}$ DC power source, provide electricity for the monitor, preheat and transfer to the video.

By connecting the power supply, and lighting the six LEDs luminescence of the prototype lens box, the image can be displayed on the monitor. The displayed images can be seen on Figure 6 . It is noted that the image shown in Figure 6 is the palm print image under other external lighting conditions.

Via testing the parameters of lens prototype in the air of $20^{\circ} \mathrm{C}, 40^{\circ} \mathrm{C}$, and in the water of $20^{\circ} \mathrm{C}$, $40^{\circ} \mathrm{C}$, respectively, the parameters of lens prototype of $40^{\circ} \mathrm{C}$ air and $40^{\circ} \mathrm{C}$ water are obtained and list in Table I and Table II, respectively. Here the temperature in cavity of the body is nearly $40^{\circ} \mathrm{C}$.

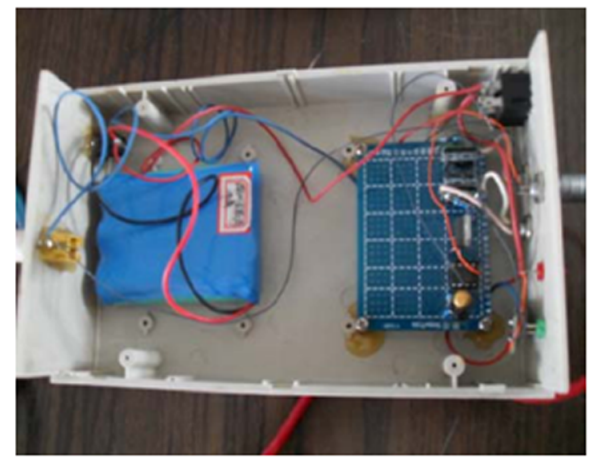

Figure 6. power box

Table 1. PARAMETERS IN $40{ }^{\circ} \mathrm{C}$ AIR

\begin{tabular}{|l|l|l|l|l|l|}
\hline Time & $\begin{array}{c}\text { Power box of } \\
\text { Voltage(V) }\end{array}$ & $\begin{array}{c}\text { Camera } \\
\text { Voltage(V) }\end{array}$ & $\begin{array}{c}\text { LED voltage } \\
(\mathrm{V})\end{array}$ & $\begin{array}{c}\text { LED Current } \\
(\mathrm{mA})\end{array}$ & $\begin{array}{c}\text { 20mm } \\
\text { Illuminance } \\
(\text { Lux })\end{array}$ \\
\hline $1 \mathrm{~min}$ & 12.59 & 4.98 & 2.56 & 45.2 & 82.4 \\
\hline $5 \mathrm{~min}$ & 12.58 & 4.98 & 2.53 & 45.3 & 82.5 \\
\hline $1 \mathrm{~h}$ & 12.36 & 4.96 & 2.57 & 45.6 & 81.3 \\
\hline $5 \mathrm{~h}$ & 12.31 & 4.97 & 2.54 & 44.6 & 80.2 \\
\hline $12 \mathrm{~h}$ & 12.13 & 4.96 & 2.49 & 46.1 & 80.3 \\
\hline $24 \mathrm{~h}$ & 11.09 & 4.98 & 2.48 & 45.1 & 80.6 \\
\hline $48 \mathrm{~h}$ & 11.05 & 4.92 & 2.48 & 46.2 & 80.8 \\
\hline $72 \mathrm{~h}$ & 11.03 & 4.96 & 2.47 & 46.1 & 80.1 \\
\hline
\end{tabular}


Table 2. PARAMETERS IN $40{ }^{\circ} \mathrm{C}$ WATER

\begin{tabular}{|l|l|l|l|l|}
\hline \multicolumn{1}{|c|}{ Time } & $\begin{array}{c}\text { Power box of } \\
\text { Voltage(V) }\end{array}$ & $\begin{array}{c}\text { Camera } \\
\text { Voltage(V) }\end{array}$ & $\begin{array}{c}\text { LED voltage } \\
(\mathrm{V})\end{array}$ & $\begin{array}{c}\text { LED Current } \\
(\mathrm{mA})\end{array}$ \\
\hline $1 \mathrm{~min}$ & 12.52 & 4.98 & 2.54 & 45.6 \\
\hline $5 \mathrm{~min}$ & 12.53 & 4.98 & 2.56 & 45.1 \\
\hline $1 \mathrm{~h}$ & 12.23 & 4.96 & 2.52 & 45.2 \\
\hline $5 \mathrm{~h}$ & 12.25 & 4.97 & 2.52 & 44.8 \\
\hline $12 \mathrm{~h}$ & 12.13 & 4.92 & 2.47 & 46.2 \\
\hline $24 \mathrm{~h}$ & 11.01 & 4.93 & 2.47 & 43.2 \\
\hline $48 \mathrm{~h}$ & 11.06 & 4.90 & 2.46 & 45.5 \\
\hline $72 \mathrm{~h}$ & 11.07 & 4.92 & 2.45 & 43.7 \\
\hline
\end{tabular}

The working stability can be seen through working parameters on the two tables. Even the intensity of illumination can not be measured in water, it can be deduced from the voltage current condition of led. From these Tables, the distinguish determination can be decided to distinguish 0.2 mm objects.

Experimenting with $1000 \mathrm{~mA}$ charger, which has been in charging for 8 hours, it can guarantee the continuous 80-hour working time. No Light Spot Front Located Hysteroscopy Camera Found no spot.

\section{Summary}

By installing the light and the video at the front, the designed hysteroscopy system can reduce its energy consuming by eliminating intermediate transmission without decreasing the image quality. The power box with a $12 \mathrm{~V} \mathrm{DC}$ input has small dimension and light weight. The entire system is easy to carry and can be operated mostly anywhere. A $12 \mathrm{~V}$ car battery with a simple connector is sufficient to turn the system to operational mode in extreme case such as disaster relief.

\section{References}

1. The 64th session of China international medical equipment exposition exhibition [M/CD].Beijing. National Medicine Lilan Group. 2010,10

2. Q. S. Zhang. Selected LED Applied Circuit [M].Beijing. mechanical industry press, 2010,2-21

3. GB50034-2004, building lighting design standard [S]

4. Instruction for YB4180 series transistor characteristics graphics instrument [Z]. Yang: Jiangsu Yangzhong Lvyang electrical equipment Co. LTD, 2004

5. W.P Na. The Selection and Application of General Integrated Circuit [M]. Beijing. people's wiley\&sons press, 2004,129-151

6. China metrology press. New Edition for Collection of Electronic Circuit [M] .Beijing. China Met 